\title{
UN PROBLEMA EN LA LÓGICA MODAL DE KRIPKE
}

\author{
Martín Rosenberg G.
}

El presente artículo tiene por objeto exponer cómo la lógica modal de Saúl Kripke, abordada principalmente en su libro Naming and Necessity, trata el concepto de propiedad esencial y las falencias o inconsistencias que esto acarrea.

Palabras clave: nombre propio, descripción definida, designador rígido, necesidad de origen.

\section{A PROBLEM IN KRIPKE'S MODAL LOGIC}

The present paper's goal is to show how Saul Kripke's modal logic uses the concept of an essential property and the weaknesses or inconsistencies that this entails for his theory, mainly presented in his book Naming and Necessity.

Keywords: proper name, definite description, rigid designator, necessity of origin.

\footnotetext{
* Universidad Andrés Bello, Santiago, Chile. Correo electrónico: martinrosenbergg@gmail.com
} 


\section{1) Kripke v/s la Teoría de la Descripción Definida}

La Lógica Modal es el estudio formal de enunciados del tipo "es necesario que" y "es posible que". Un enunciado necesario hace referencia a algún aspecto ineludible e insoslayable de un elemento. Tomemos el siguiente enunciado como ejemplo:

P1: Es necesario que todo patriota ame a su patria.

Dentro del análisis más básico que se le puede otorgar a la palabra patriota, está el amor hacia la patria. Analíticamente, en el sentido kantiano de la palabra, el amor hacia la patria es una condición esencial en todo patriota, ya que está implícito en la misma palabra. Pero esto no quiere decir, por ejemplo, que todo patriota necesariamente pertenezca a las fuerzas armadas. Y así quedaría:

P2: No es necesario que todo patriota pertenezca a las fuerzas armadas.

La adhesión o no a las fuerzas armadas se presenta como un hecho contingente o accidental del patriota, en contraposición al "amor a la patria", el cual-como vimos en P1-, se presenta como aspecto esencial de todo patriota. Por tanto, como corolario para este ejemplo del patriotismo y las fuerzas armadas, podemos decir que:

P3: Es posible que un patriota pertenezca a las fuerzas armadas.

Mediante los enunciados P1, P2 y P3 podemos darnos cuenta que las antiguas nociones aristotélicas de propiedades esenciales y accidentales están siempre presentes y juegan un papel decisivo en el estudio de la lógica modal. La determinación de una propiedad como esencial en un elemento ${ }^{1}$, en contraposición a una propiedad como accidental, es un ejercicio que le compete a la lógica modal.

1 Bien se me puede objetar que "el ser patriota" no es un elemento, sino la propiedad de un elemento (de una persona). Hay casos en los cuales las propiedades poseen aspectos esenciales, por ejemplo para "ser abuelo" (una propiedad) es necesario ser padre. En cualquier caso, en lo que resta del presente artículo me centraré en el análisis de los aspectos esenciales de los elementos físicos. 
Los objetos físicos respecto de los cuales se establecen las propiedades esenciales y accidentales, son denominados en el lenguaje mediante el uso de un nombre propio. Un nombre propio es una expresión lingüística que sirve para denotar o designar a un objeto definido, a un elemento en particular. Este objeto puede ser animado o inanimado, racional o irracional. Por lo anterior, tenemos que objetos susceptibles de ser catalogados por nombres propios son mis pertenencias, las distintas personas, porciones de géneros naturales, etc. Gracias a las expresiones deícticas o indexicales como "ese", "aquello", "este", etc., se nos permite rotular como nombres propios a artefactos y a objetos definidos de la naturaleza. A modo de ejemplo, objetos concretamente definidos y designados por un nombre propio son: $m i$ cuaderno, ese árbol, Lionel Messi, Firulais (nombre de mi perro). No es el caso de expresiones como "los lápices" ni "los caballos", ya que no designan a ningún lápiz o caballo en particular, estas son expresiones más bien genéricas.

El pilar de la filosofía kripkeana radica en la interpretación que se hace de los nombres propios. Hasta antes de Kripke, la interpretación más aceptada era la teoría descriptivista del nombre propio. Muchos renombrados filósofos contemporáneos contribuyeron o bien suscribieron a esta teoría (Frege, Russell, Wittgenstein, Searle, etc). La teoría descriptivista del nombre propio hace hincapié en la distinción entre el sentido y la referencia de un nombre. Es decir, el nombre propio no solo permite designar, referir a un objeto, sino que además le otorga un sentido al nombre mismo, y en buenas cuentas, al objeto al cual el nombre refiere. Este sentido también suele interpretarse como forma de presentación del objeto. Y este sentido dependerá del conocimiento y la experiencia que tenga el hablante con el nombre propio -al que refiere el objeto-, o bien con el objeto mismo. Por ejemplo, para nosotros tiene sentido el nombre "Aristóteles", pero no podemos elaborar sentidos respecto a Aristóteles mismo. Solo quien lo haya conocido directamente puede elaborar un sentido propio acerca de Aristóteles, como por ejemplo "el tipo que me debe dinero". Nosotros hemos de contentarnos con el nombre "Aristóteles", que refiere al autor de tales y cuales obras, alumno de tal persona, profesor de esta otra persona, y a partir de ahí elaboramos un sentido, no a partir de Aristóteles mismo, sino del uso que hacemos de su nombre.

Esta teoría descriptivista del nombre propio está muy asociada al concepto de descripción definida. Una descripción definida es una expresión lingüística que permite fijar una referencia específica en el mundo, y que además aporta un predicado respecto al objeto referido. En este predicado radica el sentido o la forma de presentación que se asocia al nombre propio. A modo de ejemplo, el nombre propio "Cecilia Bolocco" puede ser designado a través de múltiples descripciones definidas, como por ejemplo:

-La única chilena en ganar el concurso de belleza Miss Universo.

-La ganadora del concurso de belleza Miss Universo 1987.

-La segunda esposa de Carlos Menem.

-La madre del hijo menor de Carlos Menem. 
Cada una de las anteriores descripciones definidas designa específicamente a Cecilia Bolocco. Por esto son descripciones definidas; definen al objeto sobre el cual se habla además de decir algo sobre él, de entregar algún tipo de información, de describirlo, en buenas cuentas. Bien podría decirse que, en cierto sentido, la descripción definida equivale a un sinónimo del nombre propio, pero que añade algún tipo de información.

No siempre el nombre propio está asociado a una y la misma descripción definida, esto es: el objeto o el nombre que lo designa, se presenta de distintas formas. Es decir, el objeto o nombre propio puede mostrarse diferente ante distintas personas, e incluso ante la misma persona en distintos periodos de tiempo, dependiendo esto último en buena medida de las experiencias de la persona con el nombre o con el objeto mismo y de las circunstancias en las que la persona se encuentre. Esta variabilidad en las formas de presentación, cuando de nombres propios se trata, se ve reflejada sobre todo en nombres fuertemente cargados de sentido o connotación, como por ejemplo el nombre "Fidel Castro". Cuando se le pregunta a distintas personas por este nombre, las descripciones definidas son tan dispares entre sí, que pareciera que estuvieran hablando de personas ontológicamente distintas, es decir, habría más de una referencia para este nombre.

Respecto a la multiplicidad de descripciones definidas asociadas a un mismo nombre, se presenta la teoría descriptivista en su versión racimo (cluster), la cual establece que el nombre pasa a ser, metafóricamente hablando, una percha de donde se van colgando distintas descripciones definidas. Para ejemplificar la teoría descriptivista en su versión racimo, tomemos el nombre propio "Gabriela Mistral”. Descripciones definidas que se agrupan en torno a este nombre son:

-El seudónimo de Lucila Godoy Alcayaga.

-La ganadora del premio Nobel de Literatura de 1945.

-La primera mujer iberoamericana en recibir el premio Nobel, etc.

El racimo de descripciones definidas relacionado a un mismo nombre es variable de acuerdo a la persona, a su nivel cultural, a sus intereses, sus ocupaciones, experiencias, preferencias, etc. Teniendo en cuenta lo anterior, resulta imposible establecer a una descripción definida, a una propiedad, como necesaria o esencial en un objeto designado por un nombre. Esto es así debido a que cualquier juicio de este tipo sería antojadizo, subjetivo y relativo, es decir, tendría todas las cualidades antagónicas a las que en principio habría de tener una propiedad esencial o necesaria.

Esta posición anti-esencialista es uno de los principales rasgos de la teoría descriptivista del nombre propio. La teoría descriptivo-cualitativa del nombre propio niega o rechaza el concepto de esencia a través de una relativización de todas las propiedades de la entidad. De esta manera, una distinción tentativa sobre propiedades esenciales y accidentales en un objeto o nombre, se ve reducida a esquemas mentales personales, no extrapolables al resto de los hombres o a las cosas mismas. 
Martín Rosenberg G.

Kripke tiene una opinión totalmente distinta con respecto al nombre propio, si se la compara con la teoría descriptivista. Quizás el principal rasgo de la filosofía kripkeana radica en su esencialismo. Contrariamente a los filósofos descriptivistas, Kripke cree firmemente que las cosas, las entidades físicas, todas ellas, tienen esencia.

Para que las cosas a las cuales los nombres propios refieren tengan esencia, es necesario darle otro tratamiento a los nombres propios. Como ya vimos, si interpretamos a un nombre propio como una descripción definida o como un conjunto de ellas, estamos conducidos a una mirada anti-esencialista con respecto al problema. Por tanto, rechazar la teoría de la descripción definida del nombre propio es una prioridad en la filosofía de Kripke.

Para rechazar a esta teoría, Kripke introducirá la noción de designador rígido. Un “designador rígido" va a designar en todo mundo posible a un mismo elemento. Por ejemplo si digo: "en un mundo posible Diego Maradona podría haber tenido una mancha verde en la cara", el significado semántico de la expresión es claro; uno toma a Diego Maradona, el que vemos en los medios de comunicación, y le agregamos una mancha verde en la cara. De esta forma se cumpliría el requisito propuesto en el mundo posible "Diego Maradona podría haber tenido una mancha verde en la cara". Esto de "tomar a Diego Maradona del mundo real" que puede sonar muy trivial, Kripke le va a denominar estipulación, y de esta manera en los mundos posibles las entidades se estipulan. La rigidez del designador rígido se da en este sentido: mediante el uso de la estipulación la referencia se mantiene rígida.

Si alguien llegara a preguntarse lo siguiente: "pero en ese mundo posible, ¿son ese Diego Maradona y el Diego Maradona del mundo real, la misma persona?"

Kripke responde afirmativamente a esta pregunta, e incluso nos aclara el panorama, ya que si el nombre "Diego Maradona” nos genera algún tipo de dudas en este ejemplo, ¿por qué no habría de generarnos problemas la expresión "mancha verde"? Kripke argumenta que uno simplemente estipula las entidades, tanto "Diego Maradona" como "mancha verde". Uno toma estas expresiones del contexto habitual para ponerlas en una situación contrafáctica, en un mundo posible, pero las entidades son las mismas gracias a la estipulación que hacemos de ellas.

En el anterior ejemplo de Diego Maradona, de acuerdo a Kripke, nos es lícito postular una mancha verde en su cara en un mundo posible. Pero no es el caso si decimos: "en un mundo posible Diego Maradona podría haber sido un árbol", ya que esto atenta contra las propiedades ontológicas esenciales de Diego Maradona.

Tomando lo anterior en cuenta, tenemos restricciones para la estipulación de las entidades en los mundos posibles, esto es, somos libres de plantear las situaciones contrafácticas siempre y cuando estas no atenten contra las propiedades ontológicas esenciales del 
objeto en cuestión. Desde este punto de vista, todo mundo posible que uno quiera plantear respecto a una entidad, tiene una base en la realidad, y no es, en ningún caso, una situación semejante a una ficción como Alicia en el País de las Maravillas, donde nos es lícito postular prácticamente a cualquier entidad bajo cualquier propiedad.

¿Cómo se determina en cada caso, para cada entidad designada por un nombre propio, cuáles son sus propiedades ontológicas esenciales? El esencialismo kripkeano se propone responder a esta pregunta. Kripke posiciona al concepto de necesidad de origen como el encargado de guiarnos en la búsqueda de las esencias, es decir, de las propiedades que el objeto no puede dejar de tener en ningún mundo posible, ya que sin ellas dejaría de ser tal objeto. Nuestro autor señala al respecto:

Me parece que cualquier cosa proveniente de un origen diferente no sería ese objeto ${ }^{2}$.

Lo que Kripke quiere decir con esto, es que el origen o procedencia de un objeto le es esencial al mismo objeto. Las propiedades esenciales de los objetos se relacionan con la procedencia de estos mismos. El principio de la necesidad de origen es bastante amplio, ya que su aplicación depende del objeto al cual nos refiramos. Por ejemplo, las necesidades de origen de esta silla y las de Nelson Mandela son muy distintas, como veremos a continuación.

La aplicabilidad de la idea de la necesidad de origen tiene cuatro aspectos principales y dependerá del objeto en cuestión la aplicabilidad de cada aspecto:

(i) En lo que respecta a organismos vivos, su necesidad de origen remitirá a sus antecedentes orgánicos. Para los hombres serán sus progenitores, para un tomate será la semilla de la cual se origina, etc. Por ejemplo, "Alejandro Magno es hijo de Filipo II y Olimpia" es un enunciado necesariamente verdadero. En todo mundo posible donde queramos estipular a Alejandro Magno, este tendrá a los mismos padres. Kripke expone la necesidad de origen para organismos vivos de la siguiente manera:

¿Podría haber nacido la Reina-esta mujer misma-de padres diferentes de los que de hecho nació? [...] ¿Cómo podría ser esta mismísima mujer una persona que se hubiese originado a partir de otros progenitores, de un espermatozoide y óvulo enteramente diferentes? Podemos imaginar dada esta mujer, que varias cosas en su vida hubiesen cambiado: que se hubiera convertido en una mendiga, que su sangre real hubiese permanecido ignorada, y así sucesivamente. [...] Es posible que aunque hubiese nacido de estos progenitores nunca se convirtiera en reina. [...] Pero lo que es más difícil de imaginar es que hubiera nacido de padres diferentes. Me parece que cualquier cosa proveniente de un origen diferente no sería ese objeto ${ }^{3}$.

2 Kripke, S., Naming and Necessity, Ediciones de la Universidad Nacional Autónoma de México, México, 1980, p. 112.

3 Ibidem, pp. 110-112. 
Martín Rosenberg G.

Desde un punto de vista científico, la esencia de una persona radicaría en su ADN, aportado por sus progenitores. Y de un género natural, como los tomates, será el ADN genérico de esa especie de tomates -el hombre normal posee un ADN genérico de 23 pares de cromosomas-, mientras que para un tomate en específico será su ADN específico.

(ii) La necesidad del origen material es el aspecto de la teoría de la necesidad de origen que se relaciona con los objetos no biológicos, es decir, se relaciona con objetos sobre los cuales no se puede determinar su antecedente orgánico, ya que no se trata de una entidad viva. Por tanto, refiere a la constitución material de los objetos. De acuerdo a esta idea, por ejemplo "este borrador de madera" no podría haber sido hecho a partir de un trozo de madera que no sea este preciso trozo de madera:

[...] aunque podamos imaginar que hacemos una mesa a partir de otro pedazo de madera, o incluso de hielo, idéntica en apariencia a esta mesa, y aunque la hubiésemos podido colocar en esta mismísima posición en el salón, me parece que esto no es imaginar esta mesa como si estuviera hecha de madera o de hielo; sino más bien, esto es imaginar otra mesa, parecida a esta en todos sus detalles exteriores, hecha de otro pedazo de madera, o incluso de hielo ${ }^{4}$.

El ejemplo anterior es válido para una entidad no biológica en particular (un borrador de madera bien definido). Mientras que la explicación para un género de entidades no biológicas recae en la ciencia. De esta forma para un género como "el agua" su esencia será ser $\mathrm{H}_{2} \mathrm{O}$, o para "el oro" será ser el elemento atómico número 79 de la tabla periódica.

(iii) A estos dos aspectos de la necesidad de origen, para entidades biológicas y no biológicas, se les puede agregar un aspecto netamente ontológico, como es la propiedad de ser un objeto determinado:

No estoy sugiriendo que sólo sean esenciales el origen y la constitución sustancial. Por ejemplo, si el mismo trozo de madera del que fue hecha la mesa, hubiera sido convertido en un jarrón entonces la mesa nunca habría existido. Por tanto ser una mesa parece ser una propiedad esencial de la mesa ${ }^{5}$.

Esto queda más claro con el siguiente ejemplo: tomemos un anillo de oro. Si alguien está pensando que Kripke pasa por alto la forma de la joya, uno bien podría fundir ese metal, hacer otra joya (digamos, unos aretes), y desde este punto de vista la joya original no habría perdido su esencia, ya que está compuesta por el mismo oro. Kripke se resguarda de una posible acusación de esta índole mediante la propiedad esencial de ser un objeto determinado.

4 KRIPKE, S., Naming and Necessity, op. cit., p. 112.

5 Idem., p. 113. 
(iv) El último aspecto que nombraré para completar la idea de necesidad de origen denota a las relaciones esenciales. Estas son relaciones que el objeto en cuestión tiene en el mundo real con otros objetos. Se plantea la idea de relación esencial, ya que la interrelación entre ambos objetos guardaría un rasgo esencial, necesario para el objeto determinado. Por ende, esta relación también ha de ser estipulada en el mundo posible para preservar la identidad esencial del objeto. A modo de ejemplo, la relación que hay entre "polo positivo" y "polo negativo" es una relación esencial ${ }^{6}$.

A partir de los distintos aspectos de la idea de necesidad de origen, podemos notar que para Kripke nociones como "origen” o "procedencia” están en estrecha relación con el concepto "esencia".

Considero que ahora estamos en condiciones de responder a la pregunta que había quedado pendiente: ¿cómo se determina en cada caso, para cada entidad designada por un nombre, cuáles son sus propiedades ontológicas esenciales? Tomando en cuenta el concepto de necesidad de origen, dependerá de la entidad en cuestión. Pero podemos estar seguros que en la respuesta estará involucrada su procedencia o bien su materia.

\section{Una crítica funcionalista a la filosofía de Kripke}

Para Kripke, tanto clases (géneros naturales) como particulares poseen efectivamente rasgos esenciales. También vimos cómo se determinan en cada caso los rasgos esenciales específicos. La idea de designación rígida en mundos posibles (estipulación) no es difícil de comprender, ya que se acerca mucho al modo en que usamos el lenguaje cotidianamente. Toda la explicación kripkeana al tratamiento de las esencias se fundamenta en la aceptación de la idea de designador rígido. Si esta idea se rechaza, casi por necesidad se recurre a la explicación opuesta a Kripke, a la teoría descriptivista.

Sin embargo, desde mi punto de vista, independientemente de si se acepta o no la noción de designador rígido, es posible plantear interrogantes que Kripke parece pasar por alto. Kripke se conforma con dividir en dos grandes categorías a los objetos denotados por las expresiones referenciales:

(i) Los particulares, cuya determinación de esencia explica Kripke tanto para cosas vivas como inertes.

(ii) Los genéricos, como los géneros naturales, cuya determinación de esencia le compete a la ciencia.

Resulta muy curioso que Kripke haya omitido a los artefactos en su explicación so-

6 Cfr. Pérez Otero, Manuel, Esbozo de la filosofía de Kripke, Ediciones de Intervención Cultural, Barcelona, 2006, p. 162. 
bre el uso del designador rígido, ya que de ese uso se derivan situaciones problemáticas. Si bien en Naming and Necessity Kripke recurre en repetidas ocasiones a ejemplos en los cuales menciona artefactos, su mención se realiza mediante una expresión indéxica o deíctica, lo cual acarrea un tratamiento semejante al que recibe un nombre propio. De esta manera, Kripke hace uso de los artefactos para explicar su posición frente a los particulares, no para explicar su posición frente a un género o especie como bien puede ser un tipo de artefactos. Asombrosamente excluye a los artefactos o utensilios, es decir, a los objetos hechos por el hombre conforme a alguna finalidad, dentro de su análisis de los genéricos.

A continuación citaremos un extenso pasaje donde se pone de manifiesto lo que se acaba de explicar:

He aqui un atril. Una pregunta que con frecuencia ha surgido en filosofía es: ¿Cuáles son sus propiedades esenciales? ¿Qué propiedades, aparte de algunas triviales como la identidad consigo mismo, son tales que este objeto tiene que tenerlas si existe de alguna manera, y si el objeto no las tuviera no sería ese objeto?. Por ejemplo, podría ser una propiedad esencial de este atril el estar hecho de madera, y no de hielo. Tomemos solamente el enunciado más débil, que no está hecho de hielo; éste lo establecerá de manera tan vehemente, tal vez tan dramática, como sea necesario. Suponiendo que este atril esté realmente hecho de madera, ¿podría haber sido hecho este mismísimo atril desde el principio de su existencia, de hielo, digamos, de agua congelada del Támesis? Uno tiene la impresión de que no podría; aunque de hecho uno podría haber elaborado ciertamente un atril con aguas del Támesis, congelarlo mediante algún procedimiento y colocarlo exactamente aqui en lugar de esta cosa. Si alguien hubiera hecho eso, habría hecho, por supuesto, un objeto diferente; no habría sido este mismísimo atril y, asi, no tendríamos un caso en el que este mismo atril, aqui presente, había sido hecho de hielo o había sido hecho de agua del Támesis. La cuestión de si podría posteriormente, digamos dentro de un minuto, convertirse en hielo, es otra cosa. De manera que parecería, si un ejemplo como este es correcto, y esto es lo que han sostenido los defensores del esencialismo, que este atril no podría haber sido hecho de hielo; esto es, que en cualquier situación contrafáctica de la cual pudiésemos decir que este atril de alguna manera existió, tendríamos que decir también, que no estaba hecho de agua del Támesis congelada. Por supuesto que algunos han rechazado cualquier noción semejante de propiedad esencial por considerarla carente de significado. Generalmente se ha rechazado (y creo que esto es lo que diría, por ejemplo Quine) que depende de la noción de identidad a través de mundos posibles y que ésta, en sí misma, carece de significado. [...] Podemos hablar acerca de este mismísimo objeto y de si podría haber tenido ciertas propiedades que de hecho no tiene; por ejemplo, podría haber estado en otro cuarto distinto al que está, aun en este preciso momento, pero no podría haber sido hecho desde el principio de agua congelada ${ }^{7}$.

El mentado pasaje resulta muy interesante, pues en él se ilustra muy bien el tratamiento que Kripke deja de hacer con respecto a los artefactos. En la cita, el artefacto en cuestión

7 Kripke, S., “Identity and Necessity” en Valdés Villanueva, Luis, La Búsqueda del Significado, Tecnos, Madrid, 2006, pp. 116-117. 
es un atril, y para referirse a él usa la expresión “este atril”, especificándolo, denotándolo, aislándolo del resto de los objetos del mundo. Cuando se pregunta por su esencia, vemos que busca una respuesta material, de acuerdo a la idea de necesidad de origen, como efectivamente Kripke propone abordar a un particular. Pero, ¿es la materialidad la esencia de un atril? Ciertamente podría estar de acuerdo en que puede ser la esencia de ese atril, pero no de "los atriles".

Mediante la expresión "los atriles" hacemos referencia a una clase de objetos. Una analogía con los géneros naturales, quienes también se agrupan en clases, es plausible. Sin embargo, recordemos que la determinación de la esencia de un género natural cae en manos de la ciencia. Y la ciencia se ocupará de las propiedades constituyentes de la entidad para determinar su esencia.

Por otra parte, ¿es factible completar la analogía entre artefactos y géneros naturales, para establecer un trato semejante por parte de Kripke a ambas categorías? Para responder a esta pregunta es preciso tener en cuenta que las propiedades accidentales de los géneros naturales no toman parte en el concepto de esencia. Por ejemplo, es accidental que el agua tenga mal olor y apariencia verde, o bien, sea incolora e inodora. La propiedad esencial del agua es ser $\mathrm{H}_{2} \mathrm{O}$, y dependerá de si se cumple esta propiedad esencial de si es factible o no que existan propiedades accidentales, como ser verde, incolora, etc.

Las propiedades accidentales están determinadas por las propiedades esenciales del elemento. Si tenemos un artefacto, por ejemplo, un lápiz, entendemos fácilmente que es un aspecto contingente o accidental el hecho que el lápiz tenga tapa, se vea rojo y use una tinta azul. Estas son propiedades accidentales de un lápiz en específico. Pero si lo que se pretende es otorgar a los artefactos un tratamiento similar al de los géneros naturales para determinar sus esencias, nos tendremos que preguntar ¿cuál será entonces la esencia, en este caso de "los lápices"?

Todos tenemos un acercamiento vivencial y práctico con los artefactos. Esto conlleva a que siempre nos relacionamos con ellos de maneras específicas o indéxicas como "este teléfono", "mi lápiz" o "aquel computador que en unos años más construirán”. En nuestras vidas jamás aparece un artefacto, ni ningún objeto, libre de remisión alguna. Por tanto, Kripke provee una explicación para la esencia de los artefactos relacionada a nuestra cotidianeidad.

Sin embargo, desde un punto de vista muy kripkeano, podré argüir que es de sentido común el agrupar a un conjunto de objetos dados ciertos rasgos -esenciales o accidentales- que esos objetos compartan. Esto es inteligible, y de hecho así proceden los hombres. Basta con ir a una multitienda y preguntar si venden teléfonos para darnos cuenta de que la expresión "los teléfonos" agrupa a todos los objetos que sean denotados por la palabra "teléfono". Sin embargo, soy un convencido que el criterio que establece la clase "los teléfonos", es decir, la pertenencia de un objeto o no dentro de dicha clase, es un criterio 
totalmente funcional, no material, como Kripke postula que son las esencias.

Para ejemplificar mejor la primacía del aspecto funcional del artefacto, puede decirse que, en la actualidad, las jeringas están hechas mayormente de plástico (origen material), sin embargo hasta hace unas cuantas décadas estaban hechas mayormente de metal y vidrio. Bajo el criterio materialista que Kripke expone en su necesidad de origen, tendremos que decir que un tipo de jeringa guarda aspectos esenciales totalmente distintos del otro tipo de jeringa. Desde mi punto de vista, aseverar eso constituye un atentado contra el sentido común y la forma como usamos cotidianamente el lenguaje. Si en un mundo posible yo fuera mordido por una serpiente y tuviera acceso al antídoto del veneno que transmite la serpiente con su mordida, cualquiera de los dos tipos de jeringa me sería útil para proveerme la inyección. Su función prevalece por sobre su constitución material.

Bajo un análisis estrictamente aristotélico, es la causalidad final o teleológica, el para qué del objeto, lo que permite agrupar a distintos artefactos bajo un mismo nombre. Y es, por tanto, también el criterio que dictamina la esencia de un artefacto, ya que en la causalidad teleológica radica la funcionalidad del objeto.

La funcionalidad es también variable, depende del usuario en cuestión, y a veces varía en el mismo usuario, dependiendo de la situación. De esta manera, un martillo puede ser un instrumento que ayuda a colgar un cuadro en la pared, como también un arma homicida.

Mi argumento de la funcionalidad no es algo que esté contenido en el aspecto iii) de la necesidad de origen que se explicaba antes, es decir, no está incluida en el requisito de ser una cosa determinada. Esto porque la propiedad de ser una determinada cosa es rígido, mientras que es en la vida práctica donde se establecen las funcionalidades de las cosas. Si bien los artefactos están hechos conformes a una funcionalidad ideal, tanto los artefactos como las demás cosas, no están agotados en su funcionalidad. Es el hombre quien, en su contexto y conveniencia, le da funcionalidades a las cosas. Así, la funcionalidad propia del contexto práctico determina qué propiedad va a ser preponderante o esencial en una cosa. $\mathrm{Y}$ esto es algo muy variable. Solo de esta forma un martillo puede pasar a ser un arma homicida, y dejar atrás su esencia de ser un instrumento para la refacción de muebles, como plantearía Kripke en el punto iii) de su necesidad de origen. El criterio kripkeano de ser una cosa determinada es demasiado rígido, si se le compara con un enfoque funcional.

La causalidad material, es decir, la materia a partir de la cual está constituido el objeto, nos permite establecer categorías dentro de los objetos denotados por el nombre del artefacto en cuestión. Gracias a esto tenemos ropas de cuero, lana, tela etc. Todas estas ropas se diferencian entre sí dado su origen material, pero al mismo tiempo se agrupan bajo el nombre "ropa", ya que tienen la misma función en común (proveer abrigo).

La causa formal, que refiere a la forma que adopta el objeto, también nos permite 
establecer tipologías respecto a los artefactos. Hay sillas de muchas formas, con o sin respaldo, con cinco, cuatro, tres, dos y hasta una pata. Pero más allá de las particularidades de sus formas, es la funcionalidad la que permite agrupar bajo el nombre "silla" a todos estos objetos. Incluso en artefactos donde el criterio formal parece imponerse, como por ejemplo es el caso de "las pelotas", donde una forma esférica se podría plantear como necesaria, el criterio formal igualmente se ve relegado a un segundo plano en el caso de la pelota de rugby o en el caso de los niños jugando fútbol con una lata de Coca-Cola. La función que desempeña la pelota es la misma que la de la lata de Coca-Cola, y formalmente tienen escasa relación.

La materialidad que propone el enfoque kripkeano, por un lado nos deja entrever que existe alguna relación entre los aspectos esenciales de, por ejemplo, una chaqueta de cuero y un animal en específico - de donde se saca el cuero para la chaqueta-, lo cual es de cierta manera coherente. Pero por otro lado nos lleva a catalogar como cosas esencialmente distintas a una jeringa de plástico y a una jeringa de metal y vidrio, es decir, se vuelve incoherente cuando remite exclusivamente a la funcionalidad ciertos tipos de artefactos. ¿Qué criterio usará entonces Kripke para los artefactos genéricos?, ¿un criterio material como en el ejemplo de la chaqueta de cuero o uno funcional como en el de las jeringas?

Bajo un criterio material, como Kripke propone que son las esencias, ¿es legítimo establecer la pertenencia de un objeto respecto a una clase de artefactos, como por ejemplo "los encendedores"? Me parece que no. En la mayoría de los artefactos su constitución material puede variar y aun así seguir siendo el mismo artefacto. Prueba de ello es la existencia de encendedores de metal y de plástico.

Solo la causa teleológica dictamina la esencia del artefacto. Las causas aristotélicas materiales y formales pueden variar o ser distintas en cada artefacto, pero es la causa final o teleológica, el para qué del objeto, lo que permite agrupar a distintos artefactos bajo un mismo nombre. Si la noción de propiedades esenciales y contingentes (el esencialismo, en buenas cuentas) es lo que nos permite agrupar a distintos objetos bajo una clase, entonces propongo a la funcionalidad como criterio taxonómico para clasificar a distintos artefactos dentro de una misma clase.

Si bien no hemos rechazado la idea de designador rígido, podemos darnos cuenta de que el aparataje teórico-metafísico de Kripke no está en condiciones de dar una respuesta satisfactoria al problema de las esencias de artefactos, en el sentido genérico.

Ya sabemos bien que la contracara a la alternativa kripkeana radica en la teoría descriptivista. Y la teoría descriptivista, dado su carácter funcional, ofrece una solución para el problema. Procederé a dar una breve explicación de esto: la teoría descriptivista interpreta a los nombres propios, los cuales refieren a particulares, como un conjunto de descripciones, variables de persona en persona, que permiten fijar la referencia de un objeto mediante una descripción definida. Estas descripciones aluden a aspectos funcionales del 
objeto en cuestión. Las descripciones refieren a la manera que tiene el objeto de presentarse en el mundo. Así, Alejandro Magno se presenta como el hijo de Filipo II y de Olimpia, y también se presenta como el conquistador de Egipto, Babilonia y Persia, o como el alumno de Aristóteles, entre muchas descripciones que se pueden asociar a ese nombre. Alejandro Magno desempeña una función específica en cada caso (ser hijo de..., ser conquistador de..., ser alumno de...). Las descripciones se presentan como predicados en un enunciado, y es el predicado el que asigna la función específica del particular, del nombre propio de Alejandro Magno en este caso.

Conviene destacar que el criterio funcional bajo el cual la teoría descriptivista establece las esencias o no esencias, es un criterio relativo al mundo real y de facto, por tanto, absoluta e ineludiblemente relacionado con la cotidianeidad. Esta teoría ni siquiera requiere de la noción de mundos posibles. En este sentido, si bien Kripke argumenta a favor de lo intuitivo del concepto de mundo posible (estipulación), sería la teoría descriptivista la que se presentaría como más cercana al sentido común y la forma más corriente del uso del lenguaje.

Recordemos que un artefacto puede presentarse de distintas formas. Como mencioné, el martillo se puede presentar funcionalmente en el mundo como un arma homicida o como un instrumento que ayuda en la construcción de objetos. Las presentaciones o funciones del artefacto en el mundo nunca se agotan, solo se agotan si se acaba el objeto, es decir, si el objeto deja de existir o si su existencia pasa desapercibida. Desde esta perspectiva, la teoría descriptivista aborda con un carácter funcional a los objetos del mundo. Este carácter funcional no es tomado en cuenta por Kripke, quien opta por el materialismo. Y me parece legítimo plantear la polémica esencialista como una perspectiva funcional versus una perspectiva material del problema ${ }^{8}$.

¿Es concebible interpretar funcionalmente a un género natural? Se da el caso con porciones de ellos. Por ejemplo: el agua. Uno de los usos o funciones que se le puede otorgar es el de resguardar el correcto funcionamiento de un automóvil a través del enfriamiento de su motor. Por tanto, el agua desempeña una función dentro del motor. Pero no vamos a decir que esa es necesariamente la función del agua. Por cierto que un biólogo o un químico dirán algo distinto. Solo es concebible interpretar funcionalmente a un género natural completo desde un punto de vista científico. Por ejemplo, la función del agua es ser el vital elemento, la de la capa de Ozono es el atenuar cierta radiación solar, de la clorofila el generar energía para la célula y liberar $\mathrm{O}_{2}$ al ambiente, etc.

¿Es concebible interpretar funcionalmente a un particular? También se da el caso que los particulares se interpretan o usan como instrumentos o artefactos. Por ejemplo, todo 
carabinero es necesariamente una persona, solo contingentemente esta persona es carabinero. Pero cuando necesito de la ayuda de un carabinero, digamos en el caso de un asalto, lo que estoy rescatando como primordial de aquel carabinero es la función que éste tiene de repeler el asalto. Los antecedentes orgánicos del carabinero en cuestión poco o nada importan en este caso, por el contrario, su funcionalidad es insoslayable.

Es perfectamente coherente el conducirse por la vida de acuerdo a las funciones que a los particulares se les pueda otorgar. "Esa mujer es mi esposa", "aquel profesor que me reprobó", "este compañero de trabajo", "ese perro que me mordió". Todas estas expresiones singularizadoras desempeñan una función dentro de un contexto vital. Incluso los nombres propios que refieren a los particulares tienen más funciones que la de la simple referencia al particular portador del nombre. En este artículo el nombre propio "Kripke" desempeña una función específica, como es la de referir a las ideas o textos de Saúl Kripke, pero no refiere a Saúl Kripke mismo, a su persona.

Un caso muy interesante de funcionalidad, lo constituyen los nombres propios con connotaciones fuertes. El siguiente pasaje de Kripke explica su posición frente a esto:

Tengo que decir que hay algo que favorece la apreciación de Searle. Cuando escucho el nombre "Hitler" tengo un ilusorio sentimiento visceral de que es algo así como analítico que ese hombre era malo. Pero, en realidad, probablemente no es así. Hitler podría haber pasado sus días tranquilamente en Linz. En ese caso no diríamos que entonces ese hombre habría sido Hitler, pues usamos el nombre "Hitler" justo como el nombre de ese hombre, incluso cuando describimos mundos posibles. (Ésta es la noción que llamé designador rígido en la charla anterior.) Supongamos que decidimos seleccionar la referencia de "Hitler" como el hombre que logró matar más judios que ningún otro en la historia. Ésa es la manera como seleccionamos la referencia del nombre; pero en otra situación contrafáctica en la que otro hubiese ganado ese descrédito, no diríamos que en ese caso otro hombre habría sido Hitler. Si Hitler nunca hubiera llegado al poder, Hitler no habría tenido la propiedad que, según estoy suponiendo, usamos para fijar la referencia de su nombre.

Kripke parece otorgar algo de credibilidad a la teoría descriptivista (Searle) cuando reconoce asociar un sentimiento visceral hacia el nombre "Hitler". Reconoce que es como si existiera un vínculo analítico entre el nombre "Hitler" y el predicado "es malo". El término "Hitler" contendría de algún modo el concepto "maldad". La gente hace uso de vínculos de este orden en la cotidianeidad. La expresión "es un Hitler" es una evidencia de esto.

De acuerdo a las nociones que Kripke maneja de esencia, no es algo necesario o esencial el hecho que Hitler haya sido malo, y efectivamente así lo demuestra en el pasaje

9 KRIPKE, Saúl, Naming and Necessity, op. cit., p. 77. 
mediante su análisis. Sin embargo, es indiscutible que lo más importante y característico de Hitler es haber sido malo y haber matado a muchos judíos. Salvo algunos biógrafos o historiadores, en la cotidianeidad casi nadie sabe cuáles fueron los antecedentes orgánicos -esencia, según Kripke- de Hitler. Las principales características de Hitler no son anteriores al momento de la existencia del mismo Hitler, no remiten a sus padres o abuelos como Kripke da pie para pensar, sino remiten a lo que éste hizo en vida, que le otorgó tal reputación. De ahí que la gente haga uso de la expresión metafórica “es un Hitler”. En esta expresión, "Hitler" aglutina las connotaciones asociadas a una persona mala. Por tanto el nombre desempeña una función adjetiva. Si bien Adolfo Hitler no fue creado con la intención de cumplir alguna función determinada, como los artefactos son siempre hechos, la reputación que adquirió hace posible ocupar su nombre funcionalmente como un adjetivo a través de su connotación (significado), que en este caso otorga descrédito. En la expresión "es un Hitler", "Hitler" no denota a Adolfo Hitler, sino a lo que Adolfo Hitler hizo en vida, a su obra.

\section{Conclusiones}

En esta sección del artículo me parece oportuno considerar las definiciones de "esencia" que provee la Real Academia de la Lengua Española ${ }^{10}$. Creo que es ahí donde mejor se puede vislumbrar lo fundamental de la problemática acerca del tratamiento de las esencias. Tomemos en cuenta la primera definición:

D1: Aquello que constituye la naturaleza de las cosas, lo permanente e invariable de ellas.

Kripke parece apegarse a esta definición, ya que da respuestas convincentes a los aspectos que esta definición de esencia involucra. Posiciona a la ciencia como la disciplina racional que establece la naturaleza de los generales, y a través de sus ideas metafísicas, aborda los aspectos permanentes e invariables de los particulares. Bajo esta definición, Kripke da una explicación muy coherente del concepto "esencia".

La segunda definición dice:

D2: Lo más importante y característico de una cosa.

A esta interpretación de esencia parece responder la teoría descriptivista. La importancia de una cosa se determina en su existencia, en su relación con otras cosas del mundo. En las interrelaciones se establecen las funciones de los objetos. Una función puede sin problemas ser más importante que la constitución material del objeto, y por ende ser cata-

10 www.rae.es, septiembre de 2014 . 
logada de "esencial". Y lo más característico de una cosa es variable, ya que depende de la persona en relación con la cosa y la situación que viva la persona, para determinar la importancia que le otorgue a una característica específica u otra de la cosa.

El punto de vista kripkeano entra en conflicto con el descriptivista ya que cada uno entiende una cosa distinta por "esencia". Porque D1 difiere de D2, es que las posiciones parecen ser antagónicas entre sí. Quizás lo más importante y característico de una cosa no coincida con lo permanente e invariable de ella. Quizás no hay relación sinonímica entre D1 y D2, ni tampoco tratamiento homónimo. De esta manera, lo más importante y característico de Hitler es el haber sido malo y de una jeringa la función de proveer una inyección. Mientras que por otro lado, lo permanente e invariable de Hitler serán sus antecedentes orgánicos y de una jeringa sus antecedentes materiales.

Si esto es así, quizás Kripke no sea una oposición a la teoría descriptivista, ni viceversa. Más bien, entienden cosas distintas por "esencia". Y si analizamos bien el asunto, ambos puntos de vista dan explicaciones coherentes, es decir, desarrollan bien sus sistemas teóricos conforme a la definición de esencia que aceptan. Lo único que se le puede reprochar a Kripke es que no da una explicación acerca de la esencia de los artefactos genéricos, y precisamente por esto parecería acertada la teoría descriptivista, ya que provee de una explicación acerca de la palabra "esencia" para todos los particulares y generales que hay en el mundo. En este caso las esencias serán tan variables como las descripciones (funciones) asociadas al nombre u objeto.*

\section{Bibliografía}

Kripke, SAul, Naming and Necessity, Ediciones de la Universidad Nacional Autónoma de México, México, 1980.

"Identity and Necessity" en Valdés Villanueva, Luis, La Búsqueda del Significado, Tecnos, Madrid, 2006.

Nubiola, Jaime, El Compromiso Esencialista de la Lógica Modal: Estudio de Quine y Kripke, Eunsa, Pamplona, 1984.

Pérez Otero, Manuel, Esbozo de la filosofía de Kripke, Ediciones de Intervención Cultural, Barcelona, 2006.

Putnam, Hillary, "Psychological Predicates" en Capitan, W.H. \& Merrill, D.D., Art, Mind and Religion, University of Pittsburgh Press, Pittsburgh, 1967.

*Artículo recibido: 24 de septiembre de 2014. Aceptado: 30 de octubre de 2014. 
Martín Rosenberg G.

, Mind, Language and Reality, Cambridge University Press, 1975.

Quine, Wilfred V.O., From a Logical Point of View, Harvard University Press, Mass., 1953.

Salmon, Nathan, Reference and Essence, Prometheus Books, New York, 1981.

Searle, John, Nombres Propios y Descripciones, en Valdés Villanueva, Luis, La Búsqueda del Significado, Tecnos, Madrid, 2006.

www.rae.es, Septiembre de 2014. 